\title{
Tunable Optical Filter Based on Mechanically Induced Cascaded Long Period Optical Fiber Grating
}

\author{
Sunita P. Ugale and Vivekanand Mishra \\ Electronics Engineering Department, S. V. National Institute of Technology, Ichchhanath, Surat, Gujarat 395007, India \\ Correspondence should be addressed to Sunita P. Ugale; spu.eltx@gmail.com
}

Received 28 March 2013; Revised 30 May 2013; Accepted 31 May 2013

Academic Editor: Baldemar Ibarra-Escamilla

Copyright ( 2013 S. P. Ugale and V. Mishra. This is an open access article distributed under the Creative Commons Attribution License, which permits unrestricted use, distribution, and reproduction in any medium, provided the original work is properly cited.

We have proposed and demonstrated experimentally a novel and simple tunable optical filter based on mechanically induced and cascaded long period optical fiber gratings. In this filter variable FWHM and center wavelength is provided by cascading long period and ultralong period optical fiber gratings with different periods in a novel fiber structure. We report here for the first time to our knowledge the characterization of mechanically induced long period fiber gratings with periods up to several millimeters in novel multimode-single-mode-multimode fiber structure. We have obtained maximum loss peak of around $20 \mathrm{~dB}$.

\section{Introduction}

Long period fiber grating (LPFG) is the special case of fiber Bragg grating (FBG). It was first suggested by Vengsarkar and coworkers in 1996 [1]. LPFG can be formed by introducing periodic longitudinal perturbations of refractive index along the core of a single-mode fiber. It can couple light between fundamental core mode and copropagating cladding modes at specific resonance wavelength. The period of a typical LPFG ranges from $100 \mu \mathrm{m}$ to $1000 \mu \mathrm{m}$. The cladding modes of LPFG are absorbed by the polymer coating of the fiber hence, the transmission spectrum consists of a number of rejection bands at the resonance wavelengths. In contrast to the Bragg grating, LPFG does not produce reflected light and can serve as spectrally selective absorber. Therefore, it is also called transmission grating. LPFGs are relatively easy to produce. Their spectral properties, that is, resonance wavelength, bandwidth, and so forth, can be varied in a wide range. All these features make LPFG very attractive for many applications in telecommunication, laser, and sensor system.

LPFG can be induced optically or mechanically [2-4]. Optically induced gratings are permanent, whereas mechanically induced gratings are reversible. LPFGs formed by a mechanically induced technique have generated great interest due to their versatility in the process of fabrication. In these gratings, the fiber is subject to periodical stress, which results in alternated regions under compression and stretching that modulate the refractive index via the photoelastic effect. Mechanically induced long period fiber gratings (MLPFGs) induced by pressure need neither a special fiber nor an expensive writing device for fabrication. These gratings also offer advantages of being simple, inexpensive, erasable, and reconfigurable and also give flexible control of transmission spectrum.

\section{LPFG Mathematical Model}

Due to their highly multimode nature, cladding modes are located closely and multiple cladding modes can satisfy the phase matching condition, for a given index modulation of period $\Lambda$,

$$
\frac{2 \pi n_{\mathrm{eff}}^{\mathrm{co}}}{\lambda}-\frac{2 \pi n_{\mathrm{eff}}^{\mathrm{cl}}}{\lambda}=\frac{2 \pi}{\Lambda}
$$

where $n_{\mathrm{eff}}^{\mathrm{co}}$ is the effective index of the core mode and $n_{\mathrm{eff}}^{\mathrm{cl}}$ is the effective index of cladding mode.

For a given periodicity $\Lambda$, one can induce mode coupling between the fundamental mode and several different cladding modes [5], a property that manifests itself as a set of spiky losses at different wavelengths in the transmission spectrum. In design of optical filters, concatenation of gratings 
is required, and the relatively close-spaced resonance peaks of cladding modes can cause serious difficulties to generate a desired spectrum.

The coupled mode equations describe their complex amplitude, $A_{\mathrm{co}}(z)$ and $A_{\mathrm{cl}}(z)$

$$
\begin{aligned}
& \frac{d A_{\mathrm{co}}(z)}{d z}=i \kappa_{\mathrm{co}-\mathrm{co}} A_{\mathrm{co}}(z)+i \frac{s}{2} \kappa_{\mathrm{co}-\mathrm{cl}} A_{\mathrm{cl}}(z) e^{-i^{2} \delta z}, \\
& \frac{d A_{\mathrm{cl}}(z)}{d z}=i \kappa_{\mathrm{cl}-\mathrm{cl}} A_{\mathrm{cl}}(z) e^{-i^{2} \delta z}+i \frac{s}{2} \kappa_{\mathrm{cl}-\mathrm{co}} A_{\mathrm{co}}(z),
\end{aligned}
$$

where $A_{\mathrm{co}}$ and $A_{\mathrm{cl}}$ are the slowly varying amplitudes of the core and cladding modes, $\kappa_{\mathrm{co}-\mathrm{co}} \kappa_{\mathrm{cl}-\mathrm{cl}}$ and $\kappa_{\mathrm{co}-\mathrm{cl}}=\kappa^{*}{ }_{\mathrm{cl}-\mathrm{co}}$ are the coupling coefficients, $s$ is the grating modulation depth $\delta=\pi\left(\left(\left(n_{\text {eff }}^{\text {co }}-n_{\text {eff }}^{\mathrm{cl}}\right) / \lambda\right)-(1 / \Lambda)\right)$ and is the detuning from the resonant wavelength. The coupling is determined by the transverse fields of the resonant modes $E I$ and the average index of the grating $\Delta n I$

$$
\kappa_{i j}=\frac{\omega \varepsilon_{0} n}{4} \int \Delta n(r) E_{i}(r) E_{j}^{*}(r) d r .
$$

According to coupled mode theory, grating transmission is a function of coupling coefficient $\kappa_{i j}$.

Assuming that the detuning from resonant wavelength is balanced by the dc coupling, simplified expression for grating transmission is given by

$$
T(z)=\cos ^{2}(\kappa z) .
$$

Cross-coupling coefficient $\kappa$ depends on the grating index profile and field profiles of the resonant modes.

The LPFGs with periods exceeding one millimeter are called ultralong period fiber gratings (ULPFG). The long period size makes fabrication of ULPFG very easy as well as cheap. In LPFG, the core $\mathrm{LP}_{01}$ mode is coupled with cladding modes having the same symmetry, namely, $\mathrm{LP}_{0 m}$ modes [6]. Whereas in ULPFG, the coupling of the fundamental guided core mode to the cladding modes of high diffraction orders takes place [7]. The phase matching condition for a high diffraction order grating is given by the following equation:

$$
\lambda_{\text {res }}=\left(n_{\text {eff }}^{\mathrm{co}}-n_{\mathrm{eff}}^{\mathrm{cl}, m}\right) \frac{\Lambda}{N},
$$

where $\lambda_{\text {res }}$ is the resonant wavelength and $n_{\text {eff }}^{\mathrm{co}}$ and $n_{\text {eff }}^{\mathrm{cl}, m}$ are effective indexes of fundamental core mode and $m$ th cladding mode of $N$ th diffraction order, respectively. $\Lambda$ is the grating period, and $N$ is the diffraction order. $N=1$ for LPFG.

The resonant wavelength with the variation in the effective indexes of the core and cladding ignoring the dispersion effect is given by the following equation:

$$
\begin{aligned}
\lambda_{\text {res }}^{\prime}= & \left(n_{\mathrm{eff}}^{\mathrm{co}}-n_{\mathrm{eff}}^{\mathrm{cl}, m}\right) \frac{\Lambda}{N} \\
& \times\left[1+\frac{\left(\delta n_{\mathrm{eff}}^{\mathrm{co}}-\delta n_{\mathrm{eff}}^{\mathrm{cl}, m}\right) \times\left(d \lambda_{\mathrm{res}} / d \Lambda\right)}{\left(n_{\mathrm{eff}}^{\mathrm{co}}-n_{\mathrm{eff}}^{\mathrm{cl}, m}\right)^{2}}\right],
\end{aligned}
$$

where $\lambda_{\text {res }}^{\prime}$ is the resonant wavelength with variation in the effective indexes of core and cladding, and $\delta n_{\text {eff }}^{\mathrm{co}}$ and $\delta n_{\mathrm{eff}}^{\mathrm{cl}, m}$ are the effective index changes of the fundamental core mode and mth cladding mode of the $N$ th diffraction order.

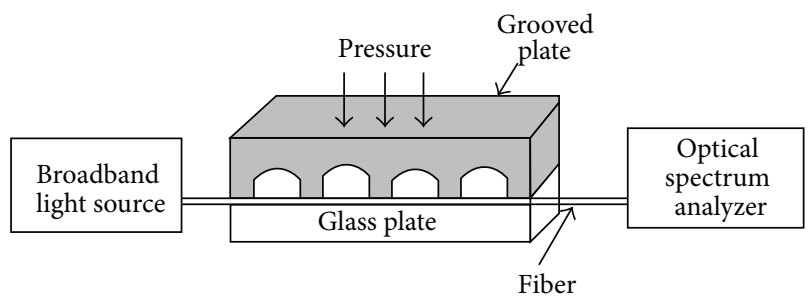

FIGURE 1: Experimental setup for inducing MLPFG and its characterization.

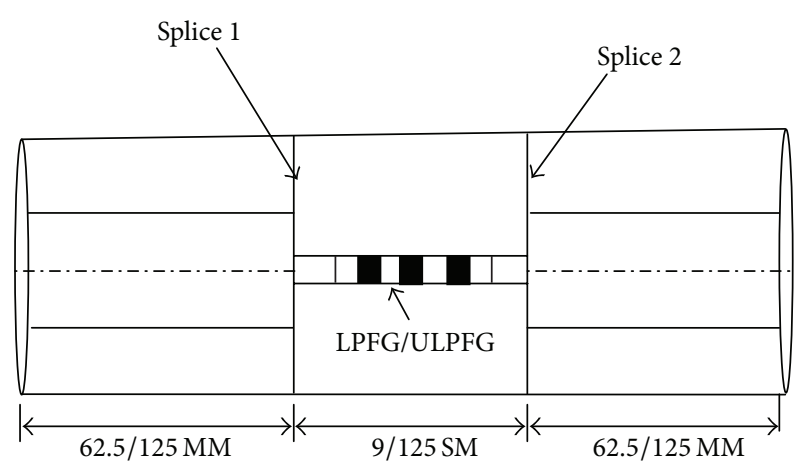

FIGURE 2: Multimode-single-mode-multimodes (MSM) fiber structure.

\section{Experiment and Results}

The MLPFG with a period of $600 \mu \mathrm{m}$ and length $=70 \mathrm{~mm}$ is induced in single-mode fiber in multimode-single-modemultimode (MSM) fiber structure $[8,9]$. Light is launched from a broadband superluminescent LED source to the lead in MMF, through the device (MLPFG) to the lead-out MMF and spectrally resolved using an optical spectrum analyzer (OSA) (Prolite60). The experimental setup as shown in Figure 1 was prepared.

A schematic diagram of the MSM structure used in experiment is shown in Figure 2. The sample is prepared by splicing a $15 \mathrm{~cm}$ long section of SMF (SMF-28) using a Sumitomo Type39 Fusion Splicer in between two MMFs $(62.5 / 125)$. Single-sided loading is done; the bottom plate is plain, while the upper plate is a specially designed grooved plate, on which pressure is applied.

The MLPFGs induced in single-mode fiber and multimode fibers are also studied separately. It is observed that single-mode grating produced resonant loss peaks of up to $\sim 7 \mathrm{~dB}$ and multimode grating produced resonant loss peaks of up to $\sim 5 \mathrm{~dB}$. In multimode fiber with a large number of core modes and cladding modes, the core-cladding power coupling occurs at many wavelengths listed in Table 1. So, many closely spaced loss peaks limit the tunable range as there are chances of overlap among different loss peaks. While in single-mode fiber, there exists only one core mode $\left(\mathrm{LP}_{01}\right)$ and many cladding modes $\left(\mathrm{LP}_{1 m}\right)$, and the core-cladding coupling occurs at certain specific wavelengths listed in Table 2.

In MSM fiber structure, while coupling from multimode fiber (core diameter $62.5 \mu \mathrm{m}$, cladding diameter $125 \mu \mathrm{m}$ ) to 


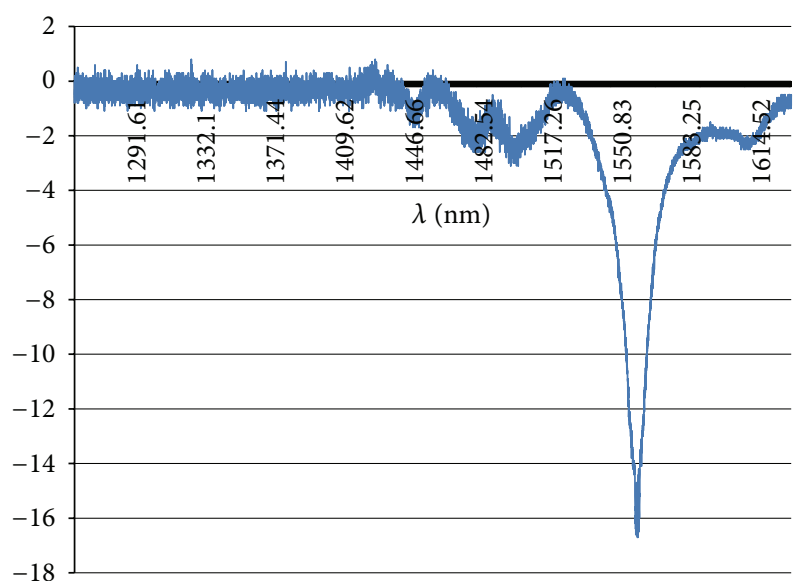

FIGURE 3: Normalized spectral response of MLPFG in MSM fiber structure.

TABLE 1: Resonance wavelengths for reversible LPFG in multimode fiber.

\begin{tabular}{llllllll}
\hline Grating period & \multicolumn{7}{c}{ Resonance wavelengths (nm) } \\
\hline $600 \mu \mathrm{m}$ & 1491 & 1505 & 1525 & 1545 & 1572 & 1597 & 1626 \\
\hline
\end{tabular}

TABLE 2: Resonance wavelengths for reversible LPFG in single-mode fiber.

\begin{tabular}{lccc}
\hline Grating period & \multicolumn{3}{c}{ Resonance wavelengths $(\mathrm{nm})$} \\
\hline $600 \mu \mathrm{m}$ & 1466 & 1510 & 1595 \\
\hline
\end{tabular}

single-mode fiber (core diameter $9 \mu \mathrm{m}$, cladding diameter $125 \mu \mathrm{m}$ ), out of many core modes in multimode fiber only one core mode gets coupled with single-mode fiber. In singlemode fiber, LPFG couples part of core mode power into a forward propagating cladding mode. In the useful communication wavelength band, the dominating coupling is taking place between core mode and the cladding mode at center resonant wavelength. Slight coupling is there with other cladding modes responsible for other loss peaks. Due to second interface between SMF and MMF, further filtering and phase shift action are taking place that will eliminate the slightly coupled wavelengths; hence, only one loss peak appears (almost at the center wavelength) in transmission spectra.

The transmission spectrum of MLPFG in MSM structure is plotted in Figure 3, and the input power spectrum is also shown for comparison purpose. The peak loss of around 17$18 \mathrm{~dB}$ is obtained, which is much greater than MLPFG in single-mode and multimode fiber.

The response of grating is very impressive and has a lot of applications in telecommunication as well as sensing. In the further section, the application of this grating is discussed.

Optical filters play important role in telecommunication systems where a certain range of wavelengths are required to be present or absent in the optical signal. Due to their low loss, compact size, high frequency response, and ease of insertion in existing optical fiber communication channels,

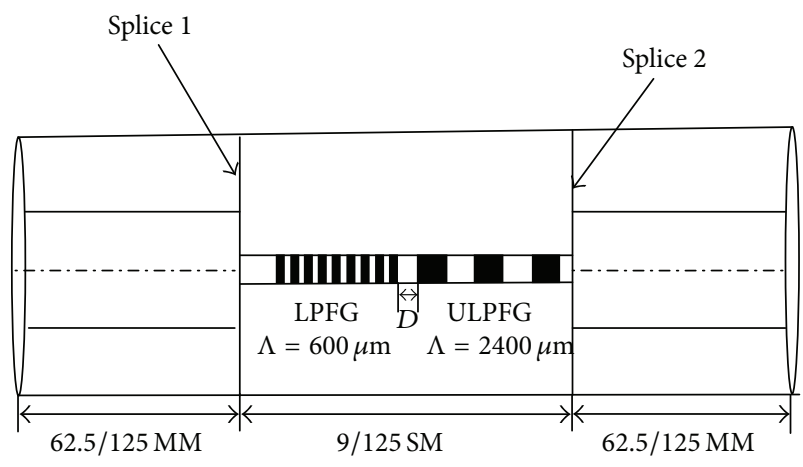

FIGURE 4: Cascading of MLPFG-MULPFG in MSM structure.

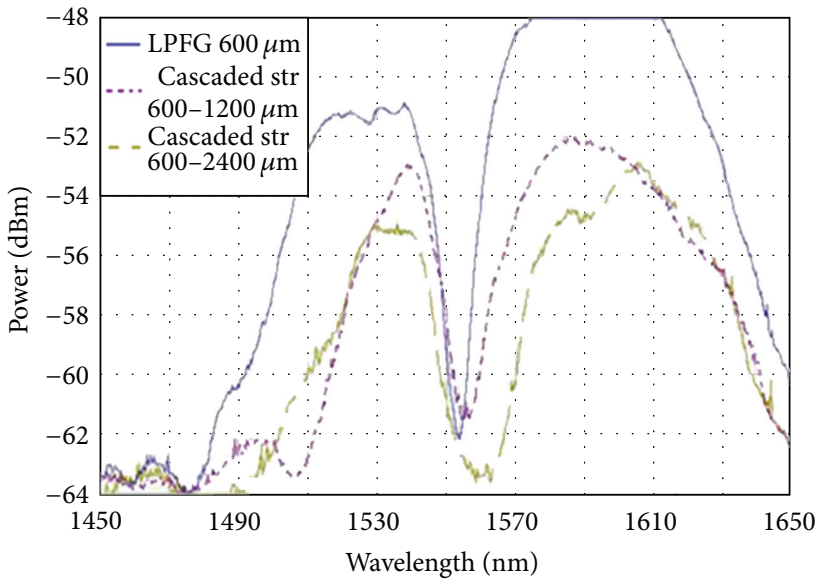

FIGURE 5: Transmission spectrums of a band-rejection filter with different gratings.

optical fiber filters are preferred over their electronic and integrated optical counterparts.

The MLPFG in MSM structure with $\Lambda=600 \mu \mathrm{m}$ and length $=70 \mathrm{~mm}$ can be used as a band reject filter. From Figure 3, the center wavelength is $1550 \mathrm{~nm}$ and the FWHM is $20 \mathrm{~nm}$. The response of grating is well within the optical spectrum used for WDM and DWCD applications (1225$1565 \mathrm{~nm}$ ), and thus the filter will be very useful in communication applications. The transmission spectrum in Figure 3 can be broadened by a novel structure of cascaded MLPFG and MULPFG shown in Figure 4.

The transmission spectrum of single MLPFG and cascaded MLPFG-MULPFG is combined and shown in Figure 5. By changing the period of gratings and the paring between gratings of different periods, the center wavelength and FWHM of filter can be changed.

\section{Conclusion}

We report here, for the first time to our knowledge, the characterization of mechanically induced LPFGs in MSM fiber structure with periods up to several millimeters. MLPFG in MSM structure gives single transmission dip. Resonant loss peak strength is around $17-18 \mathrm{~dB}$, which is much greater than 
maximum loss of $8 \mathrm{~dB}$ in single-mode MLPFG and $5 \mathrm{~dB}$ in multimode MLPFG. The MLPFG in MSM structure with $\Lambda=$ $600 \mu \mathrm{m}$ and length $=70 \mathrm{~mm}$ can be used as a band reject filter, and its center wavelength is $1550 \mathrm{~nm}$ and the FWHM is $20 \mathrm{~nm}$. The transmission spectrum obtained using single MLPFG can be broadened by a novel structure of cascaded MLPFG and MULPFG. By changing the period of gratings and the paring between gratings of different periods, the center wavelength and FWHM of filter can be changed.

\section{References}

[1] A. M. Vengsarkar, P. J. Lemaire, J. B. Judkins, V. Bhatia, T. Erdogan, and J. E. Sipe, "Long-period fiber gratings as bandrejection filters," Journal of Lightwave Technology, vol. 14, no. 1, pp. 58-65, 1996.

[2] X. Shu, L. Zhang, and I. Bennion, "Fabrication and characterisation of ultra-long-period fibre gratings," Optics Communications, vol. 203, no. 3-6, pp. 277-281, 2002.

[3] T. Zhu, Y. Song, Y. Rao, and Y. Zhu, "Highly sensitive optical refractometer based on edge-written ultra-long period fiber grating formed by periodic grooves," IEEE Sensors Journal, vol. 9, no. 6, 2009.

[4] S. Savin, M. Digonnet, G. Kino, and H. Shaw, "Tunable mechanically induced long-period fiber gratings," Optics Letters, vol. 25, no. 10, pp. 710-712, 2000.

[5] T. Erdogan, "Fiber grating spectra," Journal of Lightwave Technology, vol. 15, no. 8, pp. 1277-1294, 1997.

[6] T. Zhu, Y. J. Rao, and Q. Mo, "Simultaneous measurement of refractive index and temperature using a single ultralongperiod fiber grating," IEEE Photonics Technology Letters, vol. 17, no. 12, pp. 2700-2702, 2005.

[7] Y. Zhao and J. C. Palais, "Fiber bragg grating coherence spectrum modeling, simulation, and characteristics," Journal of Lightwave Technology, vol. 16, no. 4, pp. 554-561, 1998.

[8] J.-J. Zhu, A. P. Zhang, T.-H. Xia, S. He, and W. Xue, "Fiberoptic high temperature sensor based on thin-core fiber modal interferometer," IEEE Sensors Journal, vol. 10, no. 9, pp. 1415$1418,2010$.

[9] L. V. Nguyen, D. Hwang, S. Moon, D. S. Moon, and Y. Chung, "High temperature fiber sensor with high sensitivity based on core diameter mismatch," Optics Express, vol. 16, no. 15, pp. 11369-11375, 2008. 

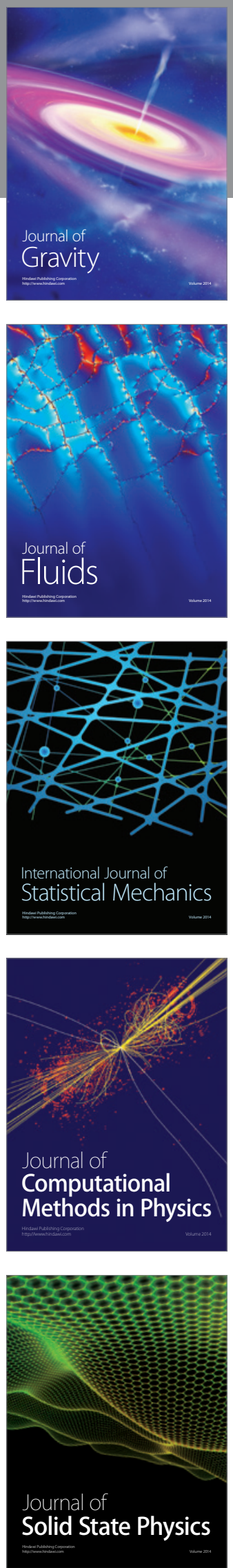

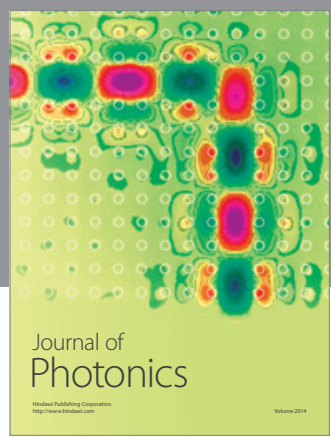

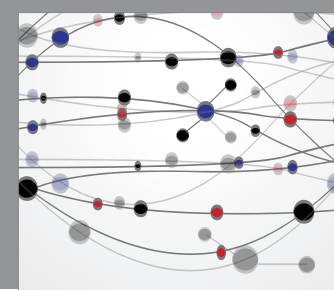

The Scientific World Journal

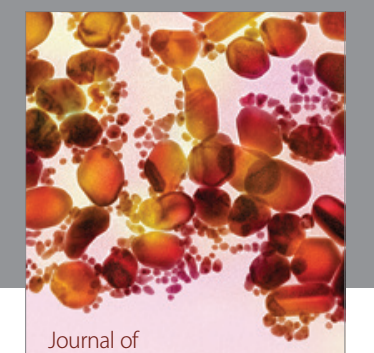

Soft Matter
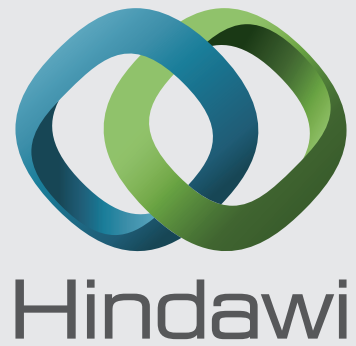

Submit your manuscripts at

http://www.hindawi.com
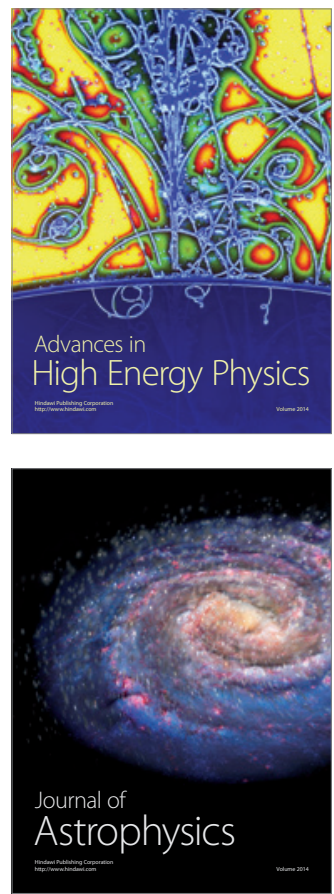
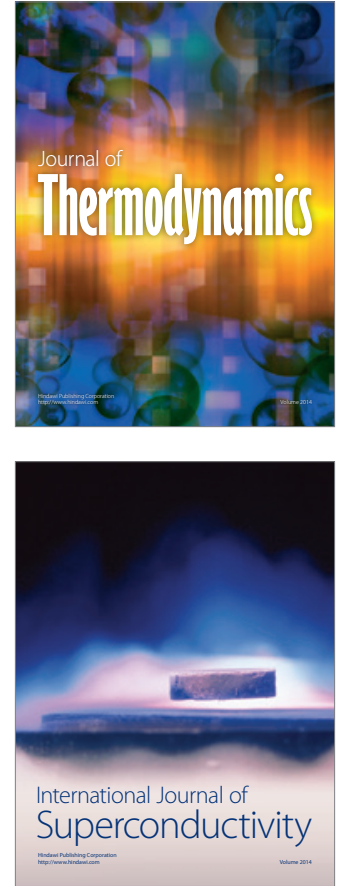
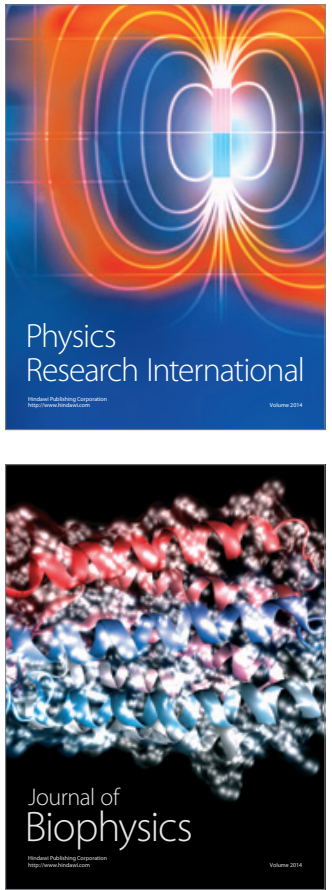
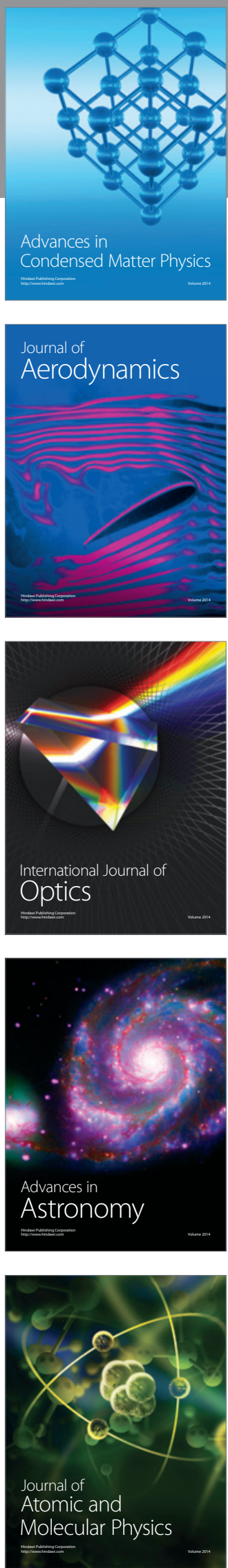